\section{Zurück zur}

\section{Reformpolitik!}

Die politischen Verheißungen der „großen Gesundheitsreformen", bei denen die jeweils neue Koalition die versammelte Berliner Gesundheits-Lobby mit hohem Pathos und dürren Eckpunkten politisch zu Tische bittet, werden inzwischen als Drohung empfunden. Die Abgeordneten konzentrieren sich längst nicht mehr auf die intensive Diskussion politischer Ziele und Anforderungen, die sie in einem Gesetzentwurf des Ministeriums erfüllt sehen wollen. Sie lassen sich stattdessen frühzeitig von der interessierten Lobby selbst mit Gesetzestechnik bewaffnen. Eingriffe, Maßnahmen und vertrackt formulierte Änderungsanträge schwellen so bald zu einem maßlosen Konvolut an Paragraphen und Begründungen an. Der Ministerialebene bleibt nur oft noch die Aufgabe, wenigstens das Schlimmste zu verhindern. Ebenso wie zu Beginn die Ziele, bleiben nun die Wirkungen weithin unklar. Im Gesundheitswesen ist jeder Millimeter komplex reguliert. Ist da der Effekt, der standatisiert wird, nicht unbekannte Folge der (vor-)letzten Intervention? Und schließlich entfaltet das Verfahren eigene Sachzwänge, mit denen es die Abgeordneten selbst überfährt. So hat kaum jemand zu guter Letzt auch nur hinreichend lesen können, was er da beschließt. Aber was rechtfertigt schon einen solchen Verzicht auf politische Kultur, zudem es natürlich wieder keine „Jahrhundertreform“ war?

Ein „Zurück zur Reformpolitik“ kann mithin nicht heißen, das gesamte Sozialgesetzbuch V mal wieder schlicht „aufzumachen“. Es braucht aber eine politische Diskussion über die vordringlichen Probleme, ihre Ursachen, Zusammenhänge und Politikoptionen. Dies umso mehr, als sich die politischen Parteien inzwischen unisono Programmpunkte wie „Patientenorientierung“, „sektorenübergreifende Versorgung“, „Teamorientierung“ und (immer wieder) „Kooperation“ zu Eigen machen. Gerade politische Postulate für die „gute Performance" eignen sich zur lobbyistischen Aneignung, solange die Politik kein klares Bild hat, wie etwa „Kooperation“ oder „Teamorientierung" gesetzestechnisch zu bewegen wären. So konnte die Lobby der aktuellen Koalition etwa andienen, es sichere die „gute Performance“ von MVZ, wenn bestimmte Rechtsformen und Trägerschaften ausgeschlossen würden. Stattdessen sollten anstelle solcher zusätzlichen, lobby-induzierten Regulierung die in den vergangenen Jahren entstandenen Verkrustungen gerade aufgelöst werden. Ein gutes Beispiel ist die Integrierte Versorgung nach $\mathbb{\$} \mathbb{S} 140 \mathrm{ff}$ SGB V. Gestartet als liberalste Rechtsgrundlage im SGB V für neue Versorgungsformen wurde die Integrierte Versorgung in den vergangenen Jahren immer weiter durchreglementiert. Folgen sind eine zunehmende Komplexität, steigender Verhandlungsaufwand für die Vertragspartner sowie Genehmigungsverfahren mit äußerst unsicherem Ausgang.

Ein „Zurück zur Reformpolitik“ kann sich mithin nicht auf das gewünschte Ergebnisse der Akteure selbst richten, sondern nur auf die dafür notwendigen staatlichen Rahmenbedingungen: Wie verhindern gesetzliche Rahmenbedingungen die „gute Versorgung“ oder machen sie erreichbar? Ermöglicht die „duale“, zweigleisig gesetzliche und private Krankenversicherung die „gute Versorgung“ oder steht sie ihr entgegen? Und welche Fragen müssen bei dem rechtlichen Übergang zu einem einheitlichen Krankenversicherungsmarkt gelöst werden? Ermöglicht die Trennung in einen ambulanten und stationären Angebotssektor die „gute Versorgung“ oder steht sie ihr entgegen? Und welche Verknüpfungen von Versorgungsplanung, Vergütungssystemen und Vertragsrechten sind für eine Modernisierung der Versorgungsstrukturen zu beachten? Welche Anforderungen und Ziele ergeben sich daraus für eine einheitliche Honorarordnung für die gesetzliche und private Krankenversicherung? Welche Anforderungen ergeben sich für die Krankenhausplanung und -finanzierung? Wie kann angesichts der ungeliebten „Spezialfachärztlichen Versorgung" der offene Zugang der Patienten zu Teams von Spezialisten doch noch ordnungspolitisch vernünftig auf die Gleise gesetzt werden? Gibt es eine Renaissance der ambulanten Praxisnetze der vergangenen 90er Jahre oder lässt sich inzwischen eine Netzwerkmedizin auf der Grundlage eines starken Leistungsverbundes als Qualitätstreiber mit Vollversorgungsanspruch herstellen? Das sind die Diskussionen und Fragen in der vorliegenden Gesundheits- und Sozialpolitik zur Bundestagswahl.

Aus diesem Stoff werden keine Stegreifprogramme erzeugt oder lobbyistische Forderungen. Die Autoren fügen hier vielmehr eine inhaltliche Diskussion zusammen, von der aus eine politische Programmatik zugespitzt werden kann. Denn primär auf die mediale und von Lobbyisten befeuerte Inszenierung sollte sich die Politik bei dieser Aufgabe nicht unbesehen stützen. Die Medienpräsenz der Themen hat häufig nichts mit ihrer Relevanz für die Bürger zu tun, wie man an dem fortgesetzt verkündeten Untergang der Pharmaindustrie, dem Ruin der Krankenhäuser, der Verarmung der Ärzte oder auch den publizierten Erfolgen von Gesundheitsreformen sehen kann. Umso erfreulicher ist, dass die politischen Stiftungen der Oppositionsparteien in jüngster Zeit sorgfältige Analysen erstellt und publiziert haben. Regierungsparteien empfinden solche Analysen offenbar eher hinderlich und wollen lieber mit Handgepäck in Koalitionsverhandlungen gehen. Und die Lobby selbst inszeniert sich in der Sorge, die Kakophonie der Interessenten verweigere ihren besonderen Anliegen die Aufmerksamkeit, von hellblau bis schlammgrau: So what!

Das Gesundheitswesen kann viel Pi-Pa-Pu erzeugen, wenn weiterhin mit (fast) jeder Reform zumindest eine zentrale Frage positiv bewegt werden kann. Ist nun die Länderblockade überwindbar, hat die Krankenhausplanung eine Chance. Lässt sich die Bürgerversicherung durchsetzen, ist die Harmonisierung der ambulanten Honorarordnungen denkbar - aber auch nur dann!

Die Herausgeber 